\title{
Extracorporeal shock wave therapy vs cryoultrasound therapy in the treatment of chronic lateral epicondylitis. One year follow up study
}

\author{
Maria Chiara Vulpiani ${ }^{1}$ \\ Sveva Maria Nusca ${ }^{1}$ \\ Mario Vetrano ${ }^{1}$ \\ Serena Ovidi ${ }^{1}$ \\ Rossella Baldini ${ }^{2}$ \\ Cristina Piermattei ${ }^{1}$ \\ Andrea Ferretti ${ }^{3}$ \\ Vincenzo Maria Saraceni ${ }^{1}$
}

1 Physical Medicine and Rehabilitation Unit, Sant'Andrea Hospital, "Sapienza" University of Rome, Rome, Italy

2 Medical Statistics, Faculty of Medicine and Psychology, Sant'Andrea Hospital, "Sapienza" University of Rome, Rome, Italy

3 Orthopaedic Unit and "Kirk Kilgour" Sports Injury Centre, Sant'Andrea Hospital, "Sapienza" University of Rome, Rome, Italy

Corresponding author:

Mario Vetrano

Physical Medicine and Rehabilitation Unit, Sant'Andrea Hospital, "Sapienza" University of Rome, Rome

Via di Grottarossa 1035-1039

00189 Rome, Italy

E-mail: mariovetrano@gmail.com

\section{Summary}

Background: the purpose of this study is to compare the therapeutic effects of extracorporeal shock wave therapy (ESWT) to those of cryoultrasound (Cryo-US) therapy in chronic lateral epicondylitis during a 12-month period.

Methods: single-blinded, randomized, controlled study of $\mathbf{8 0}$ participants treated for chronic LE with 3 ESWT sessions at 48/72-hours intervals $(n=40)$ or 12 Cryo-US therapy sessions (4 sessions per week) $(n=40)$. VAS and satisfactory results, considered as the sum of excellent and good scores in the Roles and Maudsley score, were used as outcome measures at baseline and 3, 6 and 12 months post-treatment.

Results: the results show statistically significant differences in VAS between the two groups at 6 $(p<0.001)$ and 12 months $(p<0.001)$ in favour of the ESWT Group. At 12 months, a difference of more than 2 points in the VAS between the two groups is demonstrated in favour of the ESWT Group. Considering satisfactory results, significant differences between the two groups are observed at $6(p=0.003)$ and 12 months $(p<0.001)$ in favour of the ESWT Group where patients achieve a satisfactory rate over $50 \%$.

Conclusions: ESWT has better clinical therapeutic results at 6- and 12-month follow-up as compared to Cryo-US therapy.

Level of Evidence: 1B.

KEY WORDS: elbow pain, non-operative care, physical therapies, tendinopathies.

\section{Introduction}

Lateral epicondylitis (LE), commonly called tennis elbow, is one of the most prevalent arm disorders. Its prevalence varies between 1 and $3 \%$ in the general population $^{1}$ and between 2 and $23 \%$ among occupational populations ${ }^{2}$. It's considered a process characterized by angiofibroblastic degeneration or hyperplasia within the common extensor tendon of the elbow, particularly affecting the extensor carpi radialis brevis $^{3}$. Its etiology derives from a multifactorial process, involving mechanical (repetitive/excessive mechanical loads, contusions) and structural factors (morphologic, cellular, metabolic) ${ }^{4}$. The treatments offered range from "wait and see", information and general advice, medication consisting mainly of non-steroidal anti-inflammatory drugs, to a variety of physical treatments, local corticosteroid or non-corticosteroid injections (sodium hyaluronate, botulinum toxin, prolotherapy), exercise or the use of different appliances or bandages ${ }^{5,6}$. Several studies have shown that physical modalities like pulsed magnetic fields, laser therapy, radiofrequency ablation, therapeutic ultrasound, low-intensity pulsed ultrasound (LIPUS) and extracorporeal shock waves promote tissue healing and are used in the management of tendon disorders $^{7}$. Our study has focused on two of these: extracorporeal shock wave therapy and cryoultrasound therapy. The rationale for taking into account these modalities in our study is that both treatments use sound waves, albeit at different physical properties ${ }^{8}$, 9 , and that the physical stimuli induce the response of fibroblasts ${ }^{10}$.

Extracorporeal shock wave therapy (ESWT) was introduced in Germany in the 1990's and then spread 
around the world for the treatment of musculoskeletal conditions, such as calcific tendinopathies of the shoulder, lateral epicondylitis of the elbow and plantar fasciitis ${ }^{11}$. A shock wave is a sonic pulse characterized by a initial rapid rise of a high peak pressure sometimes over $100 \mathrm{MPa}$ (1000 bar) in less than 10 ns (nanoseconds) - followed by a low tensile amplitude (up to $10 \mathrm{MPa}$ ), a short life cycle of approximately 10 microseconds and a broad frequency spectrum in the range of 16 to $20 \mathrm{MHz}^{8}$. Many studies have shown the biological/reparative effects of shockwaves in tendon pathologies through a biological mechanism called mechanotransduction, by which the tissues exposed to shockwaves convert the mechanical stimulation of the shockwaves into biochemical signals through the release of growth factors involved in neoangiogenesis, tendon proliferation and collagen synthesis ${ }^{12}$. Experimental studies ${ }^{13}$ on primary cultured human tenocytes demonstrated that ESWT enhances collagen synthesis and cell proliferation. Anyway, many of the shock-wave tissue effects are not yet completely understood and the exact mechanism of shock wave therapy needs to be completely identified.

Cryoultrasound therapy (Cryo-US) exploits the action of two different therapeutic physical modalities: cryotherapy and therapeutic ultrasound. Cryotherapy is generally regarded as a basic component of most injury management strategies and has proved its effectiveness in reducing edema and short-term pain ${ }^{14}$. Ultrasound consists of high-frequency sinusoidal sound waves ${ }^{15}$ with a peak pressure of 0.5 bar, approximately 1000 times lower than shock wave ${ }^{16}$. Therapeutic ultrasound may induce thermal and nonthermal effects in the tissues. Thermal effects include a rise in blood flow, reduction of muscle spasm, increase in the extensibility of collagen fibers and a pro-inflammatory response. Non-thermal effects are cavitation and acoustic microstreaming ${ }^{17}$ that induce stimulation of fibroblast activity, increase in protein synthesis and in blood flow, tissue regeneration, bone healing ${ }^{18}$. It has been suggested that the non-thermal effects of ultrasound are more important in the treatment of soft tissue lesions than are thermal effects ${ }^{19}$.

Cryoultrasound therapy is a new technology that could combine the benefits of therapeutic ultrasound with those of cryotherapy, which could reinforce both, avoiding the possible complications of the thermal heating effect of the ultrasound therapy and improving its mechanical and biological therapeutical effects ${ }^{20}$. In fact, a temperature decrease in deep tissues permits an enhancement in waves density in the tissue itself, increases the mechanical effect and, notably, reduces the thermic effect of the ultrasound. Recently, cryoultrasound therapy has been tested for musculoskeletal and musculotendinous disorders $20,21$.

The purpose of this single-blinded, randomized controlled clinical study is to compare the clinical therapeutic effects of ESWT and Cryo-US therapy in chronic lateral epicondylitis during a period of 12 months.

\section{Materials and methods}

\section{Patient recruitment}

Between June 2011 and January 2013, 90 patients were evaluated for eligibility and 80 patients with chronic lateral epicondylitis were included in this single-blind randomized clinical trial (Fig. 1). The criteria used for inclusion in the study were the following: age range from 18 to 75 years, clinical or instrumental diagnosis of chronic lateral epicondylitis since at least three months, intensity of pain of more than 5 on the Visual Analogue Scale (VAS) ${ }^{22}$ performing the Cozen test ${ }^{23}$, failure of previous conservative treatments, a wash-out period of at least 12 weeks since the last conservative therapy carried out before inclusion in the study, capability of filling in the questionnaires and signing the consent form. The exclusion criteria were shown in Table 1. At the time of enrolment, also taking into account inclusion and exclusion criteria, the same physician confirmed clinically the diagnosis by characteristic location of pain and tenderness to palpation at the lateral epicondyle and by pain during Cozen test. During this test the patient, with the elbow flexed, wrist radially deviated and forearm pronated, was requested to extend the wrist while the examiner palpated lateral epicondyle and resisted the patient wrist extension ${ }^{23}$. Out of 90 patients subjected to the first evaluation, 10 did not satisfy the inclusion criteria: 8 showed cervicobrachialgia, and in 2 patients a peripheral nerve entrapment syndrome ( 1 of the ulnar nerve, 1 of the posterior interosseous nerve) was suspected on the basis of the clinical examination. Therefore 80 patients were included in the study. The patients were informed in detail by an oral presentation of the scope and procedures of the study. They were then asked to participate in a clinical trial, in which they were randomly allocated to Extracorporeal Shock Wave Therapy (ESWT Group) or Cryoultrasound therapy Group (CRYO-US Group) according to a computer-generated randomization list. All patients gave written and informed consent to take part in this randomized controlled clinical study. The study protocol (Trial registration number: http://www. clinicaltrials.gov NCT02371902) was approved by Ethics and Experimental Research Committee of the Sant'Andrea Hospital, "Sapienza" University of Rome (RS:936/2012) and was carried out in accordance with the National Health Council Resolution No. 196/96. A randomized, placebo-controlled trial was not allowed. The study meets the ethical standards of Muscle, Ligaments and Tendons Journal ${ }^{24}$.

\section{Methods of treatment}

\section{ESWT Group}

ESWT was carried out by the same physician with decennial experience in focused extracorporeal shockwave therapy. A lithotripter with a cylindrical 


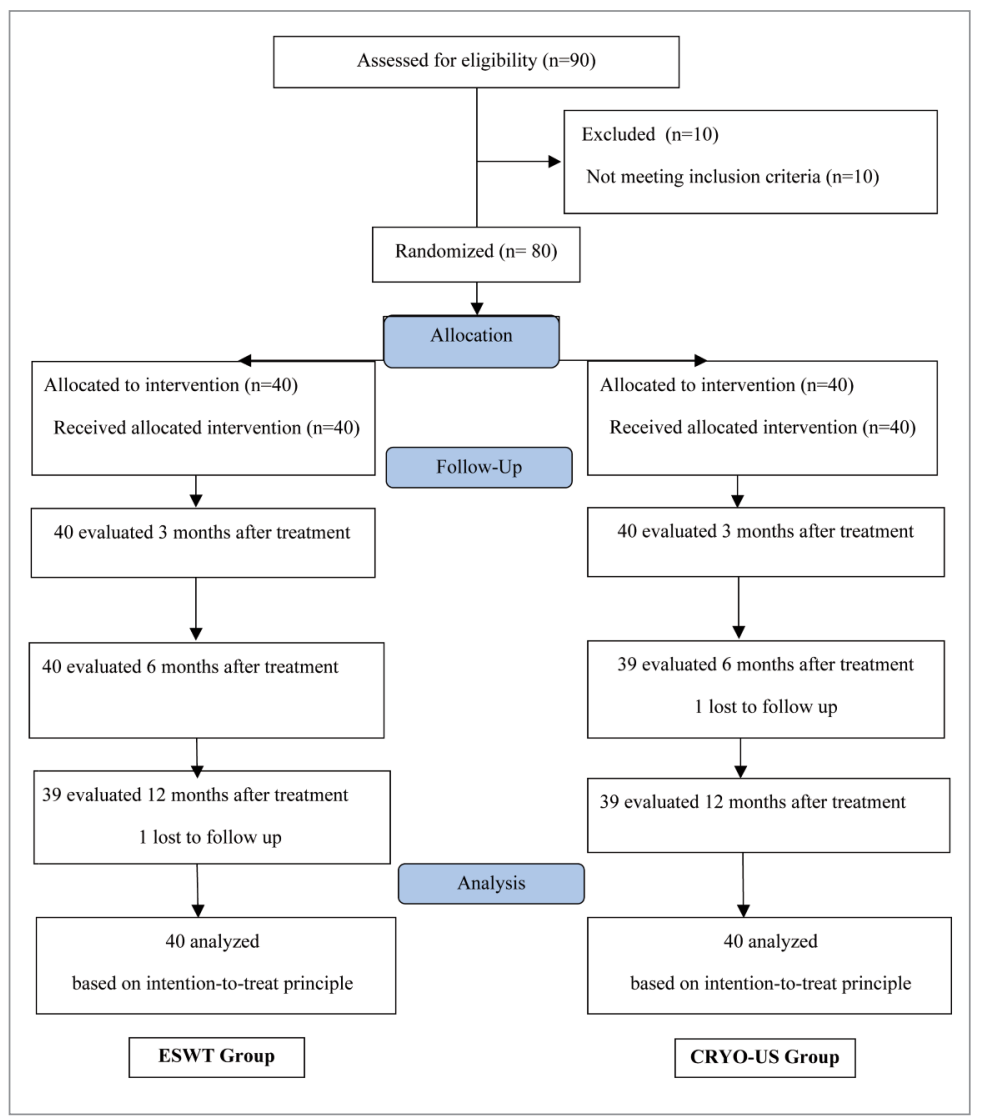

Figure 1. The CONSORT 2010 Flow Diagram.

Table 1. Exclusion criteria.

- Previous treatment with Cryo-US, ultrasound, ESW

- The conjoint presence of bilateral or lateral and medial epicondylitis

- Acute infection of the soft tissues or the bones adjacent to the area of treatment

- Local bleedings or skin lesions, pathologies of the blood coagulation, or use of anticoagulant drugs

- Pacemaker, pregnancy

- Neoplastic disease

- Raynaud's disease, altered thermal and pain sensitivity, or cold intolerance

- Evidence of elbow bursitis, or articular or synovial pathologies; signs of elbow laxity or instability

- Cervicobrachialgia; syndrome of ulnar, radial, or posterior interosseous nerve entrapment

coil electromagnetic generator (Modulith SLK, STORZ MEDICAL AG, Switzerland) was used. All patients were located in a supine position with the elbow flexed at 90 degrees. Shockwave applicator was positioned perpendicularly to the site of insertion of the wrist extensor muscles, on the lateral epicondyle. The area of treatment was identified on the basis of the locus of maximum pressure pain and by means of ultrasonic guidance, using the in-line US probe of the lithotripter. Ultrasound gel was spread over the contact point between the skin and the therapy head, in order to minimise energy dissipation at the interface between the shockwave source and the skin. Three sessions were carried out, with a time interval between sessions spanning between 48 and 72 hours. In each session, 2400 pulses were administered with energy flux density (EDF) ranging from 0.14 and $0.20 \mathrm{~mJ} / \mathrm{mm}^{2}$ depending on the maximum tolerated pain of each patient ${ }^{25}$. Analgesics or local anaesthetics were not administered before, during or after treatment.

\section{Cryo-US Group}

Treatment was carried out by the same physiotherapist with experience in cryoultrasound therapy. All patients were in a supine position with the elbow flexed at 90 degrees. A cryoultrasound equipment (Cryoultrasound $^{\mathrm{TM}}$, Medisport S.r.I., Italy) was used and the Cryo-US applicator was positioned on the lateral epicondyle over the point of maximum tenderness. A mobile head technique was used and ultrasound gel was spread over the contact point between the skin and the therapy head, in order to minimise energy dissipation at the interface between the source and the skin. Cryo-US therapy was performed in a continuous-emission modality, using an ultrasound emission power rating of $1,8 \mathrm{Watt} / \mathrm{cm}^{2}$, and a temperature of $-2^{\circ} \mathrm{C}$, for a total of 12 sessions lasting 20 minutes 
each. The treatment was performed in 3 weeks (4 sessions per week) ${ }^{20}$.

Patients of both ESWT Group and Cryo-US Group were given a standardized stretching protocol of the wrist flexor and extensor muscles (15-20 seconds $x$ 45 times) to be followed for at least 2 weeks, and subsequently they were allowed to begin usual activities if these could be performed with only mild discomfort or pain. For the time between the start and the 12months follow up patients of both groups were asked to refrain from any additional treatment for pain management and from structured therapeutic exercises.

\section{Outcome assessments}

Patients were assessed before treatment and at 3, 6 and 12 months after the end of treatments. One clinical investigator, who was blinded with regard to treatment group allocation (the blind component in the study), performed all assessments.

\section{Primary outcome}

The primary outcome was a difference of two points in pain recorded on the VAS during the Cozen test between the ESWT Group and the Cryo-US Group, in at least one of the periods taken into account. VAS is a valid and reliable measure of chronic and acute pain ranging from 'no pain' (=0 point) to 'worst imaginable pain' (=10 point) 22 . To our knowledge, Cozen test hasn't been tested for reliability and validity in LE, but it's a test widely used in clinical practice ${ }^{23}$.

\section{Secondary outcome}

The secondary outcome was defined as the number of patients who achieved at least $50 \%$ satisfactory results at 3, 6 and 12 months after the treatment. Satisfactory results were described as the sum of the excellent and good scores based on the Roles and Maudsley score 26 . This is a subjective 4-point patient assessment of pain and limitations of activity, and has been employed extensively to assess outcome after ESWT ${ }^{27}$. On this scale, 1 point is defined as an "excellent" result with the patient having no pain, full movement and full activity. Two points are defined as a "good" result, with the patient having full movement, full activity and occasional discomfort. Three points are defined as a "fair" result with the patient having some discomfort after prolonged activity. Four points indicates a "poor" outcome with patient having painlimiting activity.

\section{Statistical analysis}

To detect the difference of 2.0 points in the score of the VAS scale, an estimated standard deviation of 2.5 , with a level of significance of $5 \%$, a power of $90 \%$ and an overall correlation at follow up of 0.3 , a total of 31 subjects per group are required. Assuming a dropout of $15 \%$, at least 37 patients per group were required. As a null hypothesis, we assumed that ESWT and Cryo-US therapy have identical clinical therapeutic effects on chronic LE patients, in terms of pain reduction and satisfactory results, at 12-month follow-up. Repeated measures analysis of variance (ANOVA) was performed to assess differences in pain (VAS outcome score) across time and between treatment groups, and the interaction between time and treatment group. The $z$ test for proportions was used to compare the percentages of satisfactory results in the ESWT Group with those in the Cryo-US Group at 3, 6 and 12 months after treatment. All analyses were carried out on the basis of the intention to treat principle. A $p<0.05$ value was considered significant. The confidence interval $(\mathrm{Cl})$ at $95 \%$ was also calculated. All analyses were performed using STATA/SE 12.1 software for Windows.

\section{Results}

The baseline demographic and clinical characteristics of patients are given in Table 2. Conservative treatments previously made are NSAIDs, functional brace, rest and they are also shown in Table 2. Two patients were lost to follow-up: one of the ESWT Group did not attend the 12 month follow-up appointment, and one of the Cryo-US Group did not attend the 6 month follow-up appointment (Fig. 1). However, on the basis of the intention-to-treat, the data for these two patients were included in the analysis. We used the CONSORT 2010 Statement to flow diagram of the study $^{28}$.

\section{Primary outcome}

A significant interaction between time and treatment groups $(F=8.302 ; p<0.001)$ was showed when compared among the two groups. Significant differences between groups for the VAS score were noted at 6 months $(p<0.001)$ and 12 months $(p<0.001)$ in favour of ESWT Group. No significant difference for VAS score was found at 3 months $(p=0.130)$. At 12 months, the difference between the two treatment groups was over 2 points (Tab. 3 ).

A significant correlation between time and mean VAS score $(p<0.001)$ was noted on both ESWT Group and Cryo-US Group. Pairwise comparisons indicated in the ESWT Group a significant decrease in VAS score in all follow-ups $(p<0.001)$, whereas in the Cryo-US Group a significant decrease in pain was observed only from 0 to $3(p<0.001)$, from 0 to $6(p<0.001)$ : from 0 to 12 months $(p<0.001)$, and no significant changes were noted in the other follow-up time points (from 3 to $6(p=0.891)$, from 3 to $12(p=0.271)$, from 6 to 12 months $(p=0.216)$ (Tab. 4). Mean VAS scores in both groups at baseline and during the follow up period are represented in Figure 2. 
Table 2. Demographic and clinical characteristics of both groups.

\begin{tabular}{|c|c|c|c|}
\hline Characteristics & ESWT Group & Cryo-US Group & p Value $(95 \% ; \mathrm{Cl})$ \\
\hline Patients, no. & 40 & 40 & \\
\hline Age, mean $\pm S D$, (range), years & $49.7 \pm 9.9(25-74)$ & $53.4 \pm 10.8(32-75)$ & $0.114(-8.31 ; 0.912)$ \\
\hline \multicolumn{4}{|l|}{ Gender, no.(\%) } \\
\hline Male & $29(72.5)$ & $24(60)$ & $0.344(-0.08 ; 0.32)$ \\
\hline Female & $11(27.5)$ & $16(40)$ & \\
\hline Duration of symptoms, mean $\pm S D$, (range), months & $5.5 \pm 1.5(4-8)$ & $6 \pm 1.5(4-8)$ & $0.117(-1.17 ; 0.17)$ \\
\hline \multicolumn{4}{|l|}{ Side, no.(\%) } \\
\hline Right & $29(72.5)$ & $29(72.5)$ & $0.802(-0.19 ; 0.19)$ \\
\hline Left & $11(27.5)$ & $11(27.5)$ & \\
\hline Patients affected in their dominant arm, no. (\%) & $31(77.5)$ & $30(75)$ & $1.000(-0.16 ; 0.21)$ \\
\hline VAS on resisted extension of the wrist, mean $\pm S D$, (range), $\mathrm{cm}$ & $6.5 \pm 1.5(6-9)$ & $6.6 \pm 1.6(6-10)$ & $0.774(-0.79 ; 0.59)$ \\
\hline \multicolumn{4}{|l|}{ Previous treatments, no (\%) } \\
\hline NSAIDs & $23(57.5)$ & $22(55.0)$ & $1.000(-0.18 ; 0.23)$ \\
\hline Functional brace & $3(7.5)$ & $6(15.0)$ & $0.479(-0.22 ; 0.07)$ \\
\hline Rest & $14(35.0)$ & $12(30.0)$ & $0.811(-0.15 ; 0.25)$ \\
\hline
\end{tabular}

Abbreviations: ESWT, extracorporeal shockwave therapy; Cryo-US, cryoultrasound therapy; Cl, confidence interval; no., number; SD, standard deviation; VAS, visual analogue scale.

Table 3. Mean VAS score at baseline and 3, 6, 12 months after the end of treatment in both groups (between analysis).

\begin{tabular}{lllll}
\hline & Baseline & 3 Months & 6 Months & 12 Months \\
\cline { 2 - 5 } & Mean \pm SD & Mean \pm SD & Mean \pm SD & Mean \pm SD \\
\hline ESWT Group & $6.52 \pm 1.47$ & $4.32 \pm 2.33$ & $3.25 \pm 2.21$ & $2.32 \pm 2.25$ \\
Cryo-US Group & $6.60 \pm 1.64$ & $5.09 \pm 2.17$ & $5.15 \pm 2.19$ & $4.70 \pm 2.79$ \\
$\Delta^{\mathbf{B}}(\mathbf{9 5 \%}$ Cl) & $0.08(-0.60$ to 0.76$)$ & $0,05(-0.22$ to 1.77$)$ & $0,10(0.93$ to 2.87$)$ & $2.38(1.21$ to 3.54$)$ \\
p Value & 0,819 & 0,130 & $<0.001$ & $<0.001$ \\
\hline
\end{tabular}

Abbreviations: VAS, visual analogue scale; SD, standard deviation; ESWT, extracorporeal shockwave therapy; Cryo-US, cryoultrasound therapy; $\Delta^{\mathrm{B}}$, between-group difference; $\mathrm{Cl}$, confidence interval.

Table 4. Pairwise comparisons among mean VAS scores at all follow up time points in both groups (within analysis).

\begin{tabular}{|c|c|c|c|c|c|c|}
\hline & \multicolumn{3}{|c|}{ ESWT Group } & \multicolumn{3}{|c|}{ Cryo-US Group } \\
\hline & \multirow[b]{2}{*}{ Mean $\pm S D$} & \multirow{2}{*}{$\frac{\Delta^{\mathrm{W}}}{(95 \% \mathrm{Cl})}$} & \multirow[b]{2}{*}{$\mathrm{p}$ Value } & \multirow[b]{2}{*}{ Mean \pm SD } & \multirow{2}{*}{$\frac{\Delta^{\mathrm{W}}}{(95 \% \mathrm{Cl})}$} & \multirow[b]{2}{*}{$\mathrm{p}$ Value } \\
\hline & & & & & & \\
\hline Baseline vs 3 Month & $\begin{array}{l}6.52 \pm 1.47 \\
4.32 \pm 2.33\end{array}$ & $\begin{array}{l}-2.20 \\
(-3.07 \text { to }-1.33)\end{array}$ & 0.000 & $\begin{array}{l}6.60 \pm 1.64 \\
5.09 \pm 2.17\end{array}$ & $\begin{array}{l}-1.51 \\
(-2.47 \text { to }-0.55)\end{array}$ & 0.000 \\
\hline "” vs 6 Month & $3.25 \pm 2.21$ & $\begin{array}{l}-3.27 \\
(-4.10 \text { to }-2.44)\end{array}$ & 0.000 & $5.15 \pm 2.19$ & $\begin{array}{l}-1.45 \\
(-2.30 \text { to }-0.60)\end{array}$ & 0.000 \\
\hline " "vs 12 Month & $2.32 \pm 2.25$ & $\begin{array}{l}-4.20 \\
(-5.03 \text { to }-3.37)\end{array}$ & 0.000 & $4.70 \pm 2.79$ & $\begin{array}{l}-1.90 \\
(-2.92 \text { to }-0.88)\end{array}$ & 0.000 \\
\hline 3 Month vs 6 Month & $\begin{array}{l}4.32 \pm 2.33 \\
3.25 \pm 2.21\end{array}$ & $\begin{array}{l}-1.07 \\
(-1.79 \text { to }-0.36)\end{array}$ & 0.003 & $\begin{array}{l}5.09 \pm 2.17 \\
5.15 \pm 2.19\end{array}$ & $\begin{array}{l}0.05 \\
(-0.66 \text { to } 0.76)\end{array}$ & 0.891 \\
\hline " " vs 12 Month & $2.32 \pm 2.25$ & $\begin{array}{l}-2.00 \\
(-2.71 \text { to }-1.29)\end{array}$ & 0.000 & $4.70 \pm 2.79$ & $\begin{array}{l}-0.40 \\
(-1.11 \text { to } 0.31)\end{array}$ & 0.271 \\
\hline 6 Month vs 12 Month & $\begin{array}{l}3.25 \pm 2.21 \\
2.32 \pm 2.25\end{array}$ & $\begin{array}{l}-0.93 \\
(-1.64 \text { to }-0.21)\end{array}$ & 0.011 & $\begin{array}{l}5.15 \pm 2.19 \\
4.70 \pm 2.79\end{array}$ & $\begin{array}{l}-0.45 \\
(-1.16 \text { to } 0.26)\end{array}$ & 0.216 \\
\hline
\end{tabular}

Abbreviations: VAS, visual analogue scale; SD, standard deviation; ESWT, extracorporeal shockwave therapy; Cryo-US, cryoultrasound therapy; $\Delta^{\mathrm{w}}$ within-group difference; $\mathrm{Cl}$, confidence interval. 


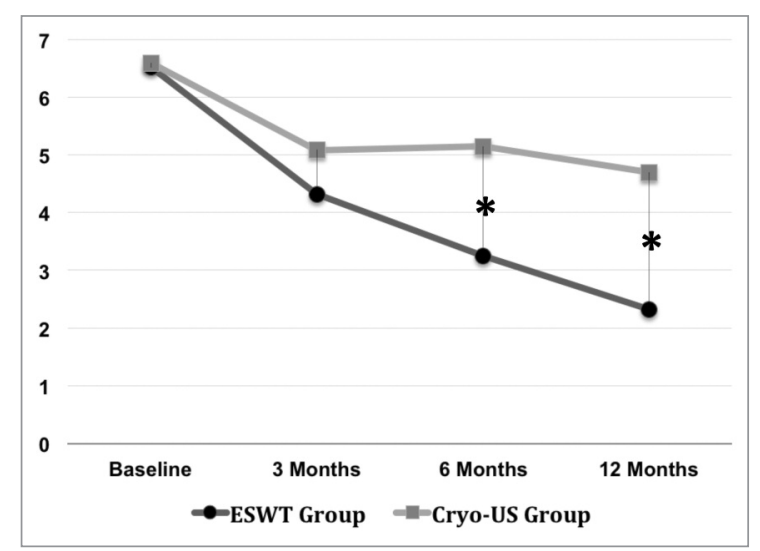

Figure 2. Line graph demonstrating mean VAS scores during the follow up period in both groups. Asterisks $\left({ }^{*}\right)$ represent significant difference between groups.

\section{Secondary outcome}

Considering the secondary outcome, the satisfaction rate required at $50 \%$ was only achieved in the ESWT Group in the follow-up at 6 (62.5\%) and 12 (70.0\%) months. Significant differences between the two groups were observed only at 6 (IC 95\% 0.13; 0.53; $\mathrm{p}=0.003$ ) and 12 months (IC 95\% $0.18 ; 0.57$; $\mathrm{p}<0.001$ ) in favour of the ESWT Group (Tab. 5).

\section{Side Effects}

All ESWT Group patients reported pain at the limit of tolerability, also due to the gradual increase in the energy level up to the values specified by the protocol. The feeling of pain ceased immediately after treatment. Moreover, the skin of the patients was examined after each session and in one third of ESWT Group patients (13/40) some blushing was noticed. Haematoma and ecchymosis were never noticed. Instead, neither side effects nor complications were noticed in Cryo-US Group patients.

\section{Discussion}

The pathophysiology of lateral epicondylitis is degen- erative in nature ${ }^{3}$. Experimental studies on animal models ${ }^{29}$, experimental researches with in vitro cell line systems ${ }^{30}$, experimental studies on primary cultured human tenocytes ${ }^{31}$ show that ESWT determines a stimulating effect on cell proliferation, as well as the activation and enhancement of the healing process. The morphological changes, proliferation and motility of treated cells, functional outcome on neovascularization and collagen synthesis, as well as the expression of differentiation clinical genes suggest that ESWT may be able to increase tendon healing ${ }^{32}$. There is strong supporting evidence from studies on animal models ${ }^{33}$ about the positive effect of ultrasound on tendon curing, despite some studies ${ }^{34}$ fail to demonstrate this. However, also some studies in vitro on animal models ${ }^{18}$ show that ultrasound can stimulate cell migration, proliferation, and collagen synthesis of tendon cells. There are no studies showing that cryoultrasound has stimulating effects on the healing process but it seems that cryoultrasound efficiency relies on the synergy between cryotherapy and ultrasound therapy, improving mechanical and biological therapeutical effects of ultrasound therapy ${ }^{20}$. In our study, short-, mid- and long-term effects of ESWT and Cryo-US on symptomatic chronic LE are compared in terms of pain reduction and patient's satisfaction. Our results do not show significant difference for VAS score and satisfaction rate between groups in the short term. Instead, there is a significant difference for VAS score and satisfaction rate in the middle and long term in favour of the ESWT group. At 12 months, a difference of more than 2 points in the VAS scale between the two groups was demonstrated in favor of shockwaves, reaching the primary outcome. At 6 and 12 months, only the ESWT group achieved a satisfactory rate over $50 \%$, reaching the secondary outcome. In the ESWT Group, there is a significant decrease in VAS score in all follow-ups compared to Cryo-US group. A recent study ${ }^{35}$ compares ESWT with US in the treatment of LE and it has found that these therapies have similar efficacy at 1-month follow-up. Instead our study compares ESWT with Cryo-US and has a longer follow up. In the short term, we have found similar results of the previous study. In the middle and long term, we have found more beneficial clinical effects of ESWT compared to Cryo-US. Unfortunately the lack of a placebo group doesn't allow us to clearly state that

Table 5. Satisfactory results at different follow-up.

\begin{tabular}{|c|c|c|c|c|}
\hline & ESWT Group $(n=40)$ & Cryo-US Group $(n=40)$ & p Value & $\mathrm{Cl} 95 \%$ \\
\hline $\begin{array}{l}\text { 3-month follow up } \\
\text { Satisfactory (excellent }+ \text { good scores) }\end{array}$ & $\begin{array}{l}\text { no, \% } \\
\text { (17) } 42.5\end{array}$ & $\begin{array}{l}\text { no, \% } \\
(14) 35.0\end{array}$ & 0.646 & $-0.13 ; 0.28$ \\
\hline $\begin{array}{l}\text { 6-month follow up } \\
\text { Satisfactory (excellent + good scores) }\end{array}$ & (25) 62.5 & (11) 27.5 & 0.003 & $0.13 ; 0.53$ \\
\hline $\begin{array}{l}\text { 12-month follow up } \\
\text { Satisfactory (excellent + good scores) }\end{array}$ & (28) 70.0 & (12) 30.0 & $<0.001$ & $0.18 ; 0.57$ \\
\hline
\end{tabular}

Abbreviations: ESWT, extracorporeal shockwave therapy; Cryo-US, cryoultrasound therapy; Cl, confidence interval; no, number of patients. 
the observed results in the 2 groups were not due to the natural course of the disease but to the effect of one of the two therapies. A randomized, placebo-controlled trial was not allowed by the Ethics Committee of our University Hospital and the lack of a placebo group represents undoubtedly the main limitation of this study. Unlike our results, a recent meta-analysis $^{36}$ shows a lack of medium- to long-term clinical benefit with nonsurgical treatments for lateral epicondylitis, included ESWT and ultrasound, when compared with simple observation or placebo. Another systematic review ${ }^{37}$ does not support the use of shock wave therapy for lateral elbow pain and a recent clinical trial ${ }^{38}$ shows no evidence of the effectiveness of ESWT versus placebo. Only some studies ${ }^{27}$ conclude that ESWT are effective in treating chronic epicondylitis. There is no firm evidence in literature from well-designed controlled studies to support the use of active ultrasound therapy for treating people with pain and musculoskeletal disorders ${ }^{39}$. Clinical studies ${ }^{40}$ show that ultrasound is no more beneficial than placebo ultrasound in lateral epicondylitis. Only few studies ${ }^{41}$ demonstrate that active ultrasound was found to be superior to placebo ultrasound in pathologies like calcific tendonitis of the shoulder. There are still very few studies ${ }^{20}, 21$ about cryoultrasound and further studies are needed to evaluate the real effectiveness of this treatment. One study ${ }^{20}$ compares Cryo-US, Laser CO2, and Tecar therapy in severe insertional tendonitis of the Achilles tendon, of the patellar tendon and of the epicondylar region. It shows that Cryo-US offers advantages in comparison with laser $\mathrm{CO} 2$ and it does not show significant differences with Tecar therapy, although it shows a better mean range of effectiveness. In later studies ${ }^{21}$ cryoultrasound therapy promises an effective and long lasting clinical improvement in patients with chronic plantar fasciitis. Our study has shown that extracorporeal shock wave offers more advantages compared to cryoultrasound therapy in the treatment of chronic lateral epicondylitis. The limitations of this study are the absence of the placebo group, the absence of a reliable, reproducible and sensitive instrument for assessment of chronic lateral elbow tendinopathy like the Patient-Rated Tennis Elbow Evaluation (PRTEE) Questionnaire 42 ; the absence of the analysis of strength of handgrip and of finger pinch; the fact that the area of treatment in Cryo-us group was identified on the basis of the locus of maximum pain location and not also by means of ultrasonic guidance, as in the ESWT group. For these reasons, further investigations are thus necessary in order to confirm our clinical results.

\section{Conclusions}

In conclusion, this study shows that ESWT has better clinical results at 6 and 12 months follow-up in comparison with Cryo-US in the treatment of symptomatic chronic LE. Additional studies are needed to confirm these observations.

\section{References}

1. Shiri R, Viikari-Juntura E, Varonen H, Heliovaara M. Prevalence and determinants of lateral and medial epicondylitis: a population study. Am J Epidemiol. 2006;164:1065-1074.

2. McCormark RR Jr, Inman RD, Wells A, Berntsen C, Imbus HR. Prevalence of tendinitis and related disorders of the upper extremity in a manufacturing workforce. J Rheumatol. 1990; 17:958-964.

3. Kraushaar BS, Nirschl RP. Tendinosis of the elbow (tennis elbow). Clinical features and findings of histological, immunohistochemical, and electron microscopy studies. J Bone Joint Surg Am. 1999;81:259-278.

4. Galloway MT, Lalley AL, Shearn JT. The role of mechanical loading in tendon development, maintenance, injury, and repair. J Bone Joint Surg Am. 2013;95:1620-1628.

5. Haahr JP, Andersen JH. Prognostic factors in lateral epicondylitis: a randomized trial with one-year follow up in 266 new cases treated with minimal occupational intervention or the usual approach in general practice. Rheumatology (Oxford). 2003;42:1216-1225.

6. Coombes BK, Bisset L, Vicenzino B. Efficacy and safety of corticosteroid injections and other injections for management of tendinopathy: a systematic review of randomized controlled trials. Lancet. 2010;376:1751-1767.

7. Sharma $P$, Maffulli N. Tendinopathy and tendon injury: the future. Disabil Rehabil. 2008:30:1733-1745.

8. Wang CJ. An Overview of shock wave therapy in musculoskeletal disorders. Chang Gung Med J. 2003;26:220-232.

9 ter Haar G. Basic physics of therapeutic ultrasound. Physiotherapy. 1978;64:100-103.

10. Frairia R, Berta L. Biological effects of extracorporeal shock waves on fibroblasts. A review. Muscles Ligaments Tendons J. 2012;1:138-147.

11. Henney JE. From the Food and Drug Administration. JAMA 2000;284:2711.

12. Notarnicola A, Moretti B. The biological effects of extracorporeal shock wave therapy (eswt) on tendon tissue. Muscles Ligaments Tendons J. 2012;2:33-37.

13. Vetrano M, d'Alessandro F, Torrisi MR, Ferretti A, Vulpiani MC, Visco V. Extracorporeal shock wave therapy promotes cell proliferation and collagen synthesis of primary cultured human tenocytes. Knee Surg Sports Traumatol Arthrosc. 2011; 19:2159-2168.

14. Bleakley C, McDonough S, MacAuley D. The use of ice in the treatment of acute soft-tissue injury: a systematic review of randomized controlled trials. Am J Sports Med. 2004;32:251261.

15. Moretti B, Amelio E, Notarnicola A. Le onde d'urto nella pratica medica. (1st edition) Bari: WIP Edizioni, 2010.

16. Ogden JA, Tóth-Kischkat A, Schultheiss R. Principles of shock wave therapy. Clin Orthop Relat Res. 2001;387:8-17.

17. Speed CA. Therapeutic ultrasound in soft tissue lesions. Rheumatology (Oxford). 2001;40:1331-1336.

18. Tsai WC, Pang GH, Hsu CC, Chu NK, Lin MS, Hu CF. Ultrasound stimulation of types I and III collagen expression of tendon cell and upregulation of transforming growth factor beta. $J$ Orthop Res. 2006;24:1310-1316.

19. Dyson M, Suckling J. Stimulation of tissue repair by ultrasound: a survey of the mechanisms involved. Physiotherapy. 1978;64:105-108.

20. Costantino C, Pogliacomi F, Vaienti E. Cryoultrasound therapy and tendonitis in athlete: a comparative evaluation versus laser CO2 and t.e.ca.r. therapy. Acta Biomed. 2005;76:37-41.

21. Costantino C, Vulpiani MC, Romiti D, Vetrano M, Saraceni VM. Cryoultrasound therapy in the treatment of chronic plantar fascitis with heel spurs. A randomized controlled clinical study. Eur J Phys Rehabil Med. 2014;50:39-47. 
22. Scott J, Huskisson EC. Vertical or horizontal visual analogue scales. Ann Rheum Dis. 1979;38:560.

23. MacDermid JC, Michlovitz SL. Examination of the elbow: linking diagnosis, prognosis, and outcomes as a framework for maximizing therapy interventions. J Hand Ther. 2006;19:82-97.

24. Padulo J, Oliva F, Frizziero A, Maffulli N. Muscles, Ligaments and Tendons Journal. Basic principles and recommendations in clinical and field science research. MLTJ. 2013;4:250-252.

25. Vetrano M, Castorina A, Vulpiani MC, Baldini R, Pavan A, Ferretti A. Platelet-rich plasma versus focused shock waves in the treatment of jumper's knee in athletes. Am J Sports Med. 2013;41:795-803.

26. Roles NC, Maudsley RH. Radial tunnel syndrome: resistant tennis elbow as a nerve entrapment. J Bone Joint Surg Br. 1972;54:499-508.

27. Rompe JD, Decking J, Schoellner C, Theis C. Repetitive lowenergy shock wave treatment for chronic lateral epicondylitis in tennis players. Am J Sports Med. 2004;32:734-743.

28. Moher D, Hopewell S, Schulz KF, et al. CONSORT 2010 Explanation and Elaboration: Updated guidelines for reporting parallel group randomised trials. J Clin Epidemiol. 2010;63:e137.

29. Chen YJ, Wang CJ, Yang KD, et al. Extracorporeal shock waves promote healing of collagenase-induced Achilles tendinitis and increase TGF-beta1 and IGF-1 expression. J Orthop Res. 2004;22:854-861.

30. Chao YH, Tsuang YH, Sun JS, et al. Effect of Shock Wave on tenocyte proliferation and extracellular matrix metabolism. UItrasound Med Biol. 2008;34:841-852.

31. Leone L, Vetrano M, Ranieri D, et al. Extracorporeal Shock Wave treatment (ESWT) improves in vitro functional activities of ruptured human tendon-derived tenocytes. PloS One. 2012;7:e49759.

32. Visco V, Vulpiani MC, Torrisi MR, Ferretti A, Pavan A, Vetrano $M$. Experimental studies on the biological effects of extracor- poreal shock wave therapy on tendon models. A review of the literature. Muscles Ligaments Tendon J. 2014;4:357-361.

33. Gan BS, Huys S, Sherebrin MH, Scilley CG. The effects of ultrasound treatment on flexor tendon healing in the chicken limb. J Hand Surg Br. 1995;20:809-814.

34. Larsen A, Kristensen G, Thorlacius-Ussing O, Oxlund $H$. The influence of ultrasound on the mechanical properties of healing tendons in rabbits. Acta Orthop. 2005;76:225-230.

35. Vural M, Diracoglu D, Erhan B, Gunduz B, Ozhan G, Pekedis K. Efficacy of extracorporeal shock wave therapy and ultrasound treatment in lateral epicondylitis: A prospective, randomized, controlled trial. Annals of Physical and Rehabilitation Medicine. 2014;57:e190.

36. Sayegh ET, Strauch RJ. Does Nonsurgical Treatment Improve Longitudinal Outcomes of Lateral Epicondylitis Over No Treatment? A Meta- analysis. Clin Orthop Relat Res. 2015;473:10931107.

37. Buchbinder R, Green SE, Youd JM, Assendelft WJ, Barnsley L, Smidt N. Shock wave therapy for lateral elbow pain. Cochrane Database Syst Rev. 2005;4:CD003524.

38. Staples MP, Forbes A, Ptasznik R, Gordon J, Buchbinder R. A randomized controlled trial of extracorporeal shock wave therapy for lateral epicondylitis (tennis elbow). J Rheumatol. 2008;35:2038-2046.

39. Robertson VJ, Baker KG. A review of therapeutic ultrasound: effectiveness studies. Phys Ther. 2001;81:1339-1350.

40. Lundeberg T, Abrahamsson P, Haker E. A comparative study of continuous ultrasound, placebo ultrasound and rest in epicondylalgia. Scand J Rehabil Med. 1988;20:99-101.

41. Ebenbichler GR, Erdogmus CB, Resh KL, et al. Ultrasound therapy for calcific tendonitis of the shoulder. N Engl J Med. 1999;340:1533-1538.

42. Rompe JD, Overend TJ, MacDermid JC. Validation of the Patient-rated Tennis Elbow Evaluation Questionnaire. J Hand Ther. 2007;20:3-10. 